\title{
Constrained Unsharp Masking for Image Enhancement
}

\author{
Radu Ciprian Bilcu and Markku Vehvilainen \\ Nokia Research Center, Visiokatu 1, 33720, Tampere, Finland \\ radu.bilcu@nokia.com, markku.vehvilainen@nokia.com \\ http://research.nokia.com/ ^bilcu
}

\begin{abstract}
In this paper we present a cost-effective solution for combined de-noising and sharpening of digital images. Our method combines the unsharp masking and sigma filtering techniques through a regularization mechanism thus ensuring effective noise reduction and edge enhancement in the processed image. We describe our method in detail and we analyze the proposed implementation through extensive experiments done in various scenarios. Due to its low computational complexity the proposed method is well suited for mobile implementations.
\end{abstract}

Keywords: Image sharpening, de-noising, unsharp masking.

\section{Introduction}

Digital photography has gained an increased interest, in the mobile devices industry, during the last decade. It is a common fact nowadays that mobile devices, such as mobile phones and PDA's, are equipped with digital cameras able to capture images and video clips. Due to their specific hardware limitations the quality of the captured images are not yet at the level of the SLR (single-lens reflex) cameras. The resolution of the imaging sensors, used in the cameraphones increases, but the total sensor size is limited, which decreases the active area of a sensor pixel. As a consequence, the captured images have a lower signal to noise ratio. Miniaturization and cost limitations enforce the use of lower quality optical systems in the cameraphones compared with the ones used in the SLR cameras. This very often introduces blur in the captured images and video clips. The low signal to noise ratio and the optical blur are probably the most important distortions that must be corrected by an imaging chain designed to be implemented in cameraphones. A very large amount of research has been done in the field of image de-noising and sharpening in order to address these two important problems and various solutions have been proposed in the open literature. Mobile devices possess a very limited processing power and contain several applications that must run in parallel which decreases even more the resources allocated for image processing tasks. As a consequence, there is a large interest in the development of low complexity image processing solutions suitable for mobile implementations. 
In this paper we propose a cost effective solution to the problem of combined de-noising and sharpening of digital images. Our proposed method combines the sigma filtering and unsharp masking (USM), through a simple regularization step, to accomplish both noise reduction and edge enhancement. The reason why we have selected these two techniques is due to their relatively good performances obtained at a very low computational cost. The paper is organized as follows: in Section 2 several related existing sharpening methods are briefly reviewed. In Section 3 we introduce our proposed approach and we illustrate its functionality through simple experiments done on synthetic images. In Section 4 we show the results of our method obtained for real images and Section 5 concludes the paper.

\section{Existing Approaches}

The problem of image sharpening has been extensively studied and many approaches have been proposed in the open literature. Bilateral filter have been used in [13. to implement a combined method for sharpening and de-noising. The novelty of this method consists in the introduction of an offset term in the range filter part of the bilateral filter. This offset is adapted to the local characteristics of the input image such that the behavior of the algorithm changes from smoothing to edge enhancement according to the local characteristics of the image. Although its good performances, this algorithm needs some optimization of the parameters which is done by training on a set of images. Image sharpening has been implemented also by means of fuzzy networks [10, using methods modeling the human visual system [6], by non-linear filtering in transform domain [7] or using diffusion filtering [3], to mention a few. Although their good performance, the above mentioned approaches have some characteristics that makes them to be quite difficult to be implemented in low power devices. For instance the method in 10 make use of 2 fuzzy networks connected in parallel and have longer processing time compared with other approaches. The nonlinear methods from [6] and 7], despite their excellent performance, have also relatively large computational complexity. The coupled shock-diffusion filtering method from 3] is expected to have also a long processing time since it is an iterative process.

Despite its simplicity, the unsharp masking technique 9] provides relatively good performance in enhancing the image sharpness thus representing a good alternative for practical implementations. In the unsharp masking technique the high frequency content of the input image is emphasized as depicted in Fig. 7. According to this figure the input image is filtered by a high-pass filter (the dotted block denoted as HPF). The high-pass filtered image $I_{H}(i, j)$ is then multiplied by a constant parameter $\lambda$ and added to the input image. There are several possibilities to implement the HPF filter. We should mention here that Fig. 7 suggests to implement the HPF filtering as the difference between the input image and its smoothed version (the output of the low-pass filter LPF). Equally well this operation can be implemented by a single high-pass convolution kernel [9]. Despite its simplicity and good performance, in many applications, the unsharp masking technique has two main drawbacks. First of all the noise present 
in the input image is amplified due to the presence of the high-pass filter. While this might not be visually disturbing in image regions with high activity (large number of edges), on smooth image areas noise amplification can give unpleasant visual distortions. Another disadvantage of the unsharp masking technique is the presence of the overshooting artifacts. This is due to a too large amplification of the high contrast areas. Among several solutions, proposed to eliminate or at least reduce these two disadvantages, the adaptive unsharp masking shown to be very effective [9]. The main idea of this method is to use a variable parameter $\lambda$ which is adaptive to the image local contrast. In this manner the regions with medium contrast are enhanced more while the smooth regions and those with high contrast are less enhanced. However, in [9], modification of $\lambda$ is done by means of an adaptive filter which implies matrix inversion and increased number of parameters to setup.

Another way to reduce the overshooting in the unsharp masking is to introduce clipping of the overshoots to the nearest local extremes [11, 8]. Some of the techniques that integrates clipping necessitate edge detection in order to be able to locate the position of the overshoots. In this paper we show that clipping not only the edge pixels, but every pixels from the image, one could obtain both de-noising and edge sharpening. We propose a modified processing scheme that combines sigma smoothing, unsharp masking and clipping to reach our goals.

\section{Proposed Approach}

In this section we describe our proposed method for combined de-noising and sharpening. In order to introduce our method we start from the block diagram

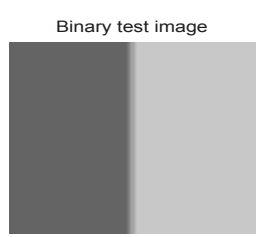

Fig. 1. Test image
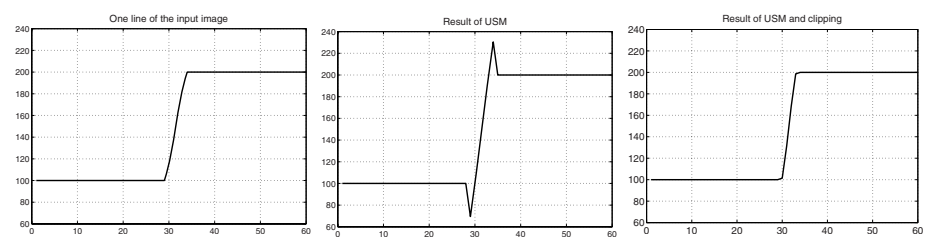

Fig. 2. One line from Fig. 3. Enhanced by test image

\section{USM}

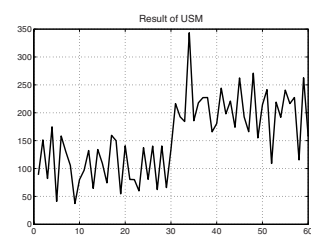

Fig. 5. One noisy line from test image
Fig. 6. Enhanced by USM 


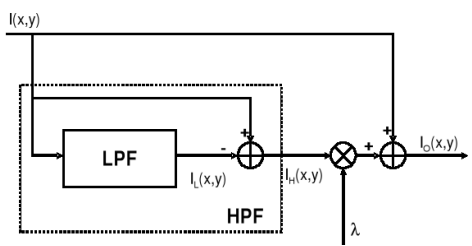

Fig. 7. Block diagram of the unsharp masking

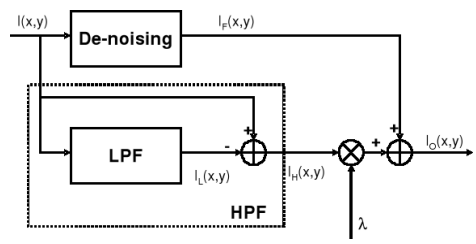

Fig. 9. Block diagram of the integrated de-noising and sharpening without clipping

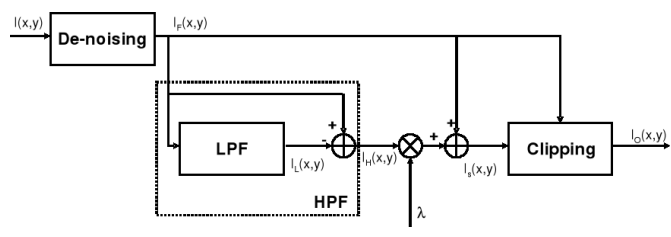

Fig. 8. Block diagram of the cascaded denoising and sharpening with clipping

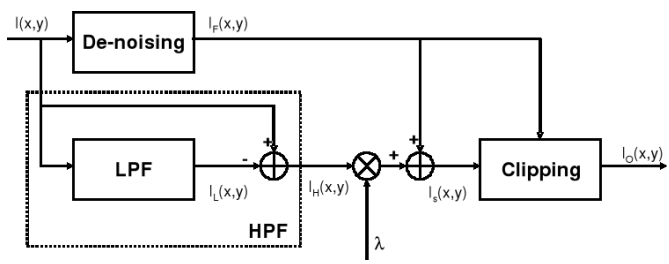

Fig. 10. Block diagram of integrated de-noising and sharpening with clipping

of the USM depicted in Fig. 7. Due to the presence of the high-pass filter (HPF) the processed image $I_{O}(i, j)$ might contain overshooting distortions around sharp edges. This effect is illustrated in Fig. 2 and Fig. 3, In Fig. 2 one line from the binary image of Fig. 1 is shown and in Fig. 3 the result of USM is depicted. These overshooting distortions can be eliminated by clipping them to the extreme

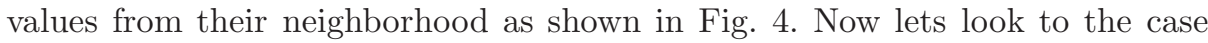
of noisy input images. To this end in Fig. 5 the same line of the binary image, with added zero mean Gaussian noise, is shown. In Fig. 6 we show the enhanced output using USM. One can notice, from this figure, the overshooting and noise amplification effects. Analyzing the plots from Fig. 5 and Fig. 6. we can observe that clipping would not be very effective in the noisy case. Indeed, the local extremes around overshoots are affected by the input noise and clipping them using the noisy local extremes could introduce artifacts. From this observation we can conclude that clipping would be more effective if it is made according to a noise-free version of the input image instead of the original noisy one. As a consequence we modify the block diagram of the USM with clipping as shown in Fig. 10. Moreover instead of clipping only edge pixels we perform clipping for all image pixels. Clipping is done according to the smoothed image $I_{F}(i, j)$. In this manner in smooth areas the noise level from the processed image is limited to the level of the remaining noise in $I_{F}(i, j)$. As a consequence, the noise in the output image will be at most equal with the noise level from $I_{F}(i, j)$ and the edges of $I_{O}(i, j)$ will be enhanced without overshooting. 

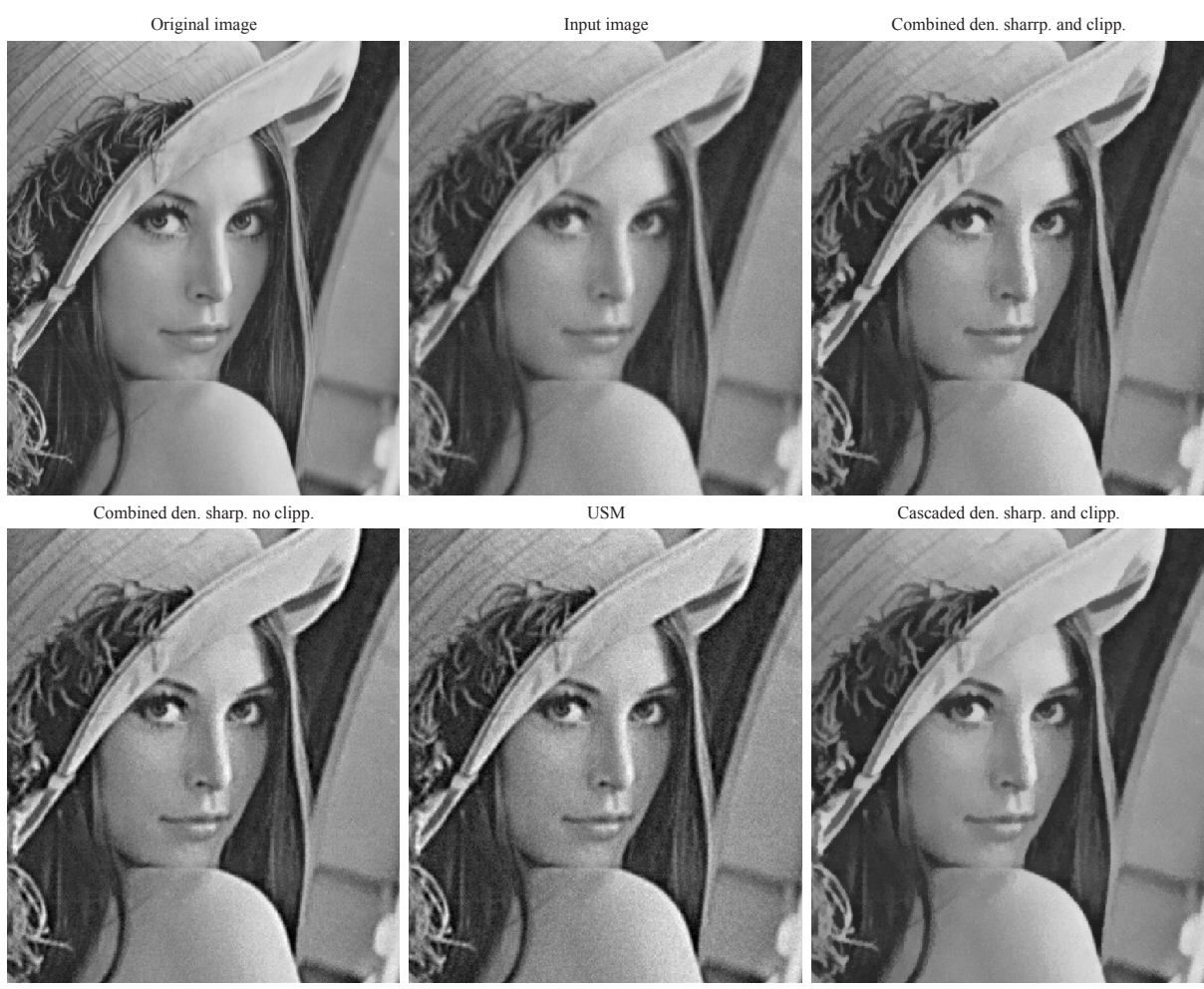

Fig. 11. From left to right and up to bottom (part of the images): Original image, noisy blurred input image, the result of the implementation from Fig. 10 the result of the implementation from Fig. 9, the result of USM (Fig. 7), the result of the implementation from Fig. 8

According to Fig. 10, the flow of our proposed algorithm is as follows:

1. Compute the image $I_{F}(i, j)$ by smoothing the input image $I(i, j)$. The smoothing process should retain as many image details as possible while reducing the input noise. Due to its simple implementation we have selected for this step the sigma filtering method [4. Other more complex image de-noising methods can be implemented as well if there is enough computational power available (see for instance [1] and the references therein).

2. Compute the image $I_{H}(i, j)$ by high-pass filtering of the input image $I(i, j)$. This can be done by convolving $I(i, j)$ with a high pass kernel $g(k, l)$.

$$
I_{H}(i, j)=(I \otimes g)(i, j)
$$

Another alternative to obtain $I_{H}(i, j)$ is to compute it as the difference between the original image $I(i, j)$ and its low-pass version $I_{L}(i, j)$ as follows:

$$
I_{H}(i, j)=I(i, j)-I_{L}(i, j), I_{L}(i, j)=(I \otimes h)(i, j) .
$$

with $h(i, j)$ being a low pass filtering kernel. 


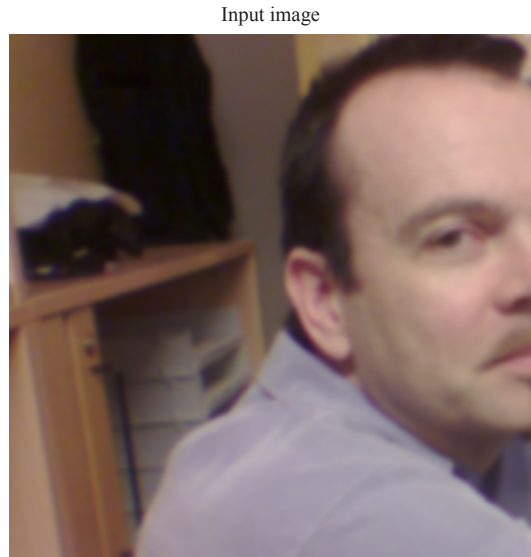

USM

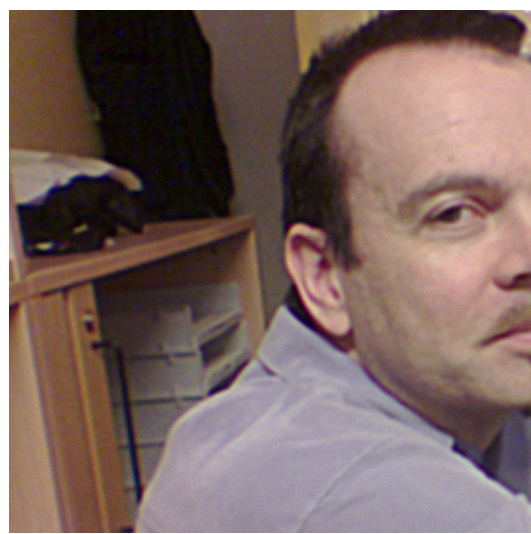

Combined den. sharp. and clipp.

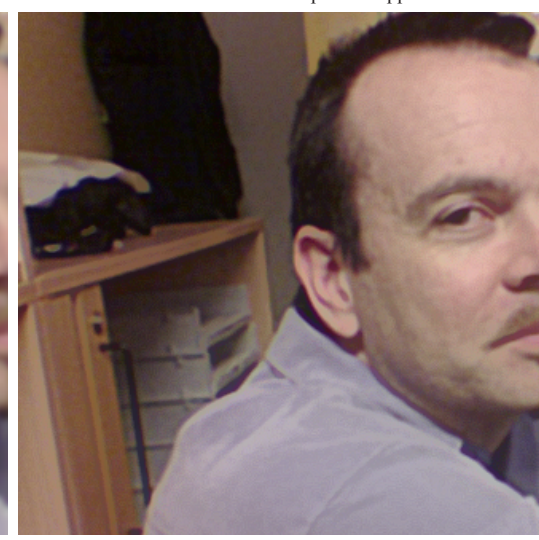

Cascaded den. sharp. and clipp.

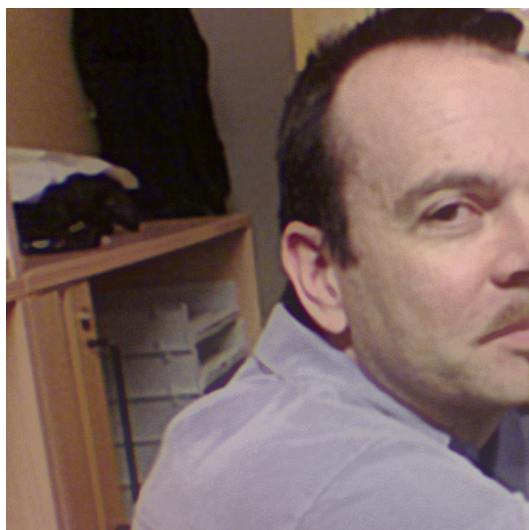

Fig. 12. From left to right and up to bottom (part of the images): original image captured by a cameraphone, the result of the implementation from Fig. 10 the result of USM (Fig. 17), the result of the method shown in Fig. 8

3. Add the enhanced high-pass image $I_{H}(i, j)$ to the de-noised image $I_{F}(i, j)$ :

$$
I_{S}(i, j)=I_{f}(i, j)+\lambda I_{H}(, j) .
$$

where $\lambda$ is a constant parameter controlling the strength of sharpening.

4. Clipping the enhanced image $I_{S}(i, j)$ according to the local extremes from $I_{F}(i, j)$ :

$$
I_{O}(i, j)=\left\{\begin{array}{l}
I_{S}(i, j) \text { if } I_{S}(i, j) \in[M I N, M A X] \\
M I N \text { if } I_{S}(i, j)<M I N \\
M A X \text { if } I_{S}(i, j)>M A X
\end{array}\right.
$$




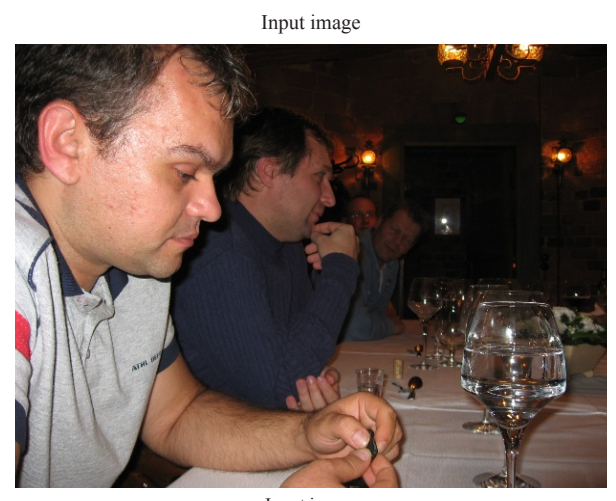

Input image

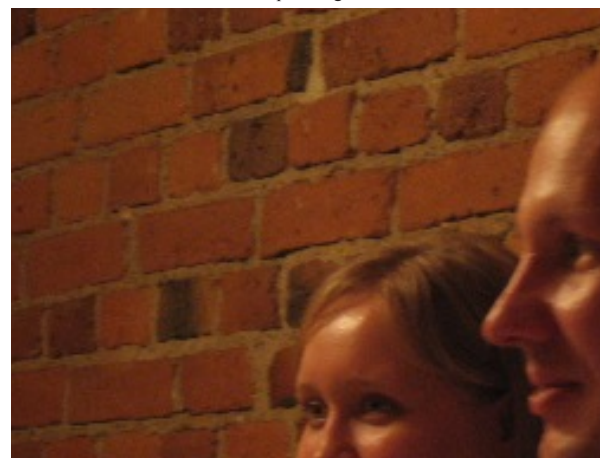

Result proposed

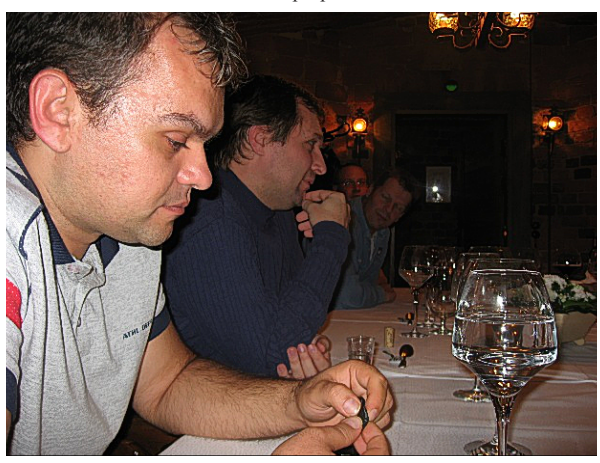

Result proposed

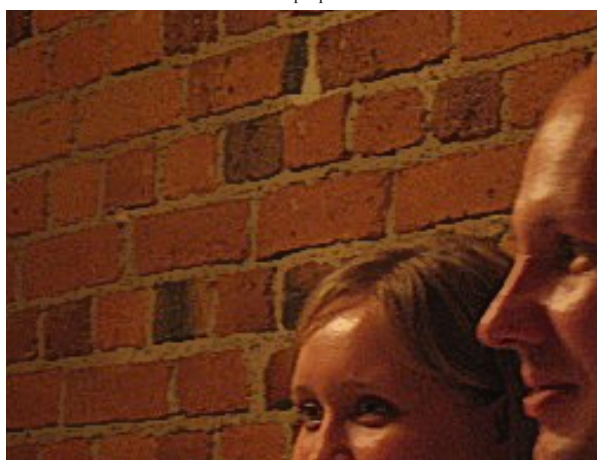

Fig. 13. Original image captured by a cameraphone (left column) and the result of the implementation from Fig. 10 (right column)

The $M I N$ and $M A X$ values are obtained from a $T \times T$ rectangular window $\Omega_{T \times T}$ centered at position $i, j$ in $I_{F}(i, j)$.

Another alternative implementation is depicted in Fig. 8. However there are differences between the method illustrated in Fig. 10 and the method depicted in Fig. 8. In the first method the high-pass filtered image $I_{H}(i, j)$ is computed from the input image while in Fig. 8 it is computed from the de-noised image $I_{F}(i, j)$. De-noising will smooth also some details of the image such that cascading denoising and USM as in Fig. 8 those details might not be enough enhanced. On the contrary, the details that would be smoothed in the de-noising process are still enhanced in Fig. 10 due to the fact that $I_{H}(i, j)$ is obtained from the original image. Since the output $I_{O}(i, j)$ is obtained by adding the enhanced high frequency content to the de-noised image $I_{F}(i, j)$, in both methods, some under enhancement of small details can be expected. However, in implementation shown in Fig. 10 this effect is somehow reduced due to the fact that $I_{H}(i, j)$ is computed from the input image. Anyway, both implementations have the same computational complexity and they perform similar in most of the cases. 
Input image

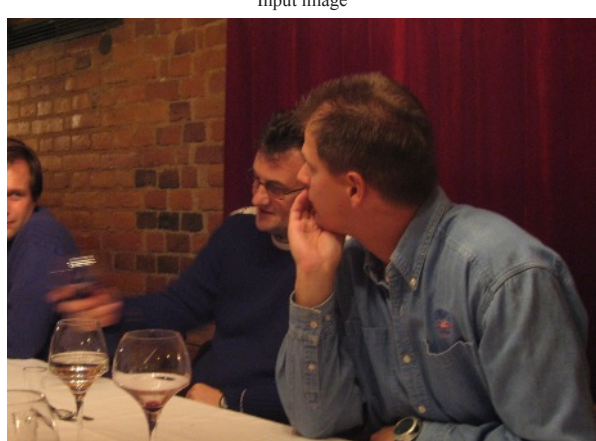

Input image

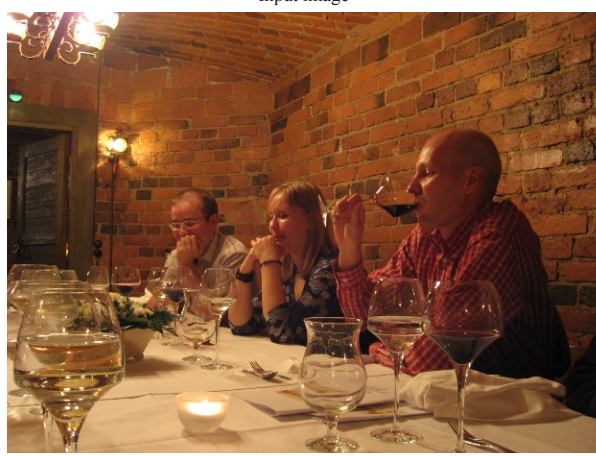

Result proposed

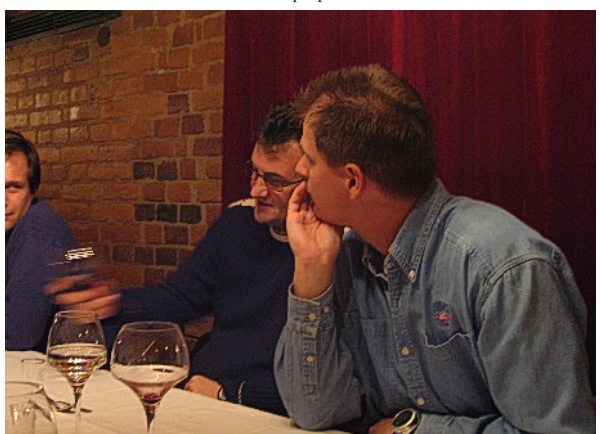

Result proposed

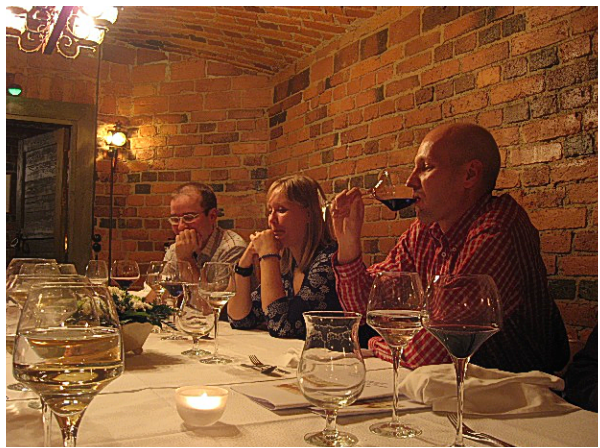

Fig. 14. Original image captured by a cameraphone (left column) and the result of the implementation from Fig. 10 (right column)

\section{Experiments and Results}

In this section we present experimental results showing the performance of our proposed approach on gray scale and color digital images. We have performed extensive experiments to verify the behavior of our new method on several well known test images as well as on images captured with cameraphones and dedicated digital cameras. The image data-base, which we have used in our experiments, contains more than 80 gray scale and color images captured with different imaging devices.

In the first set of experiments, presented here, the 8 bit image Lena was first blurred using a Gaussian kernel with $\sigma_{G}^{2}=1$ and then a zero mean Gaussian distributed noise with variance $\sigma_{n}^{2}=25$ was added to it. In Fig. 11 the processed images by the methods described in Fig. 7, Fig. 8, Fig. 9 and Fig. 10 are shown. We can see that both implementations from Fig. 8 and Fig. 10 enhance the image sharpness without increasing the noise level. This is due to the clipping mechanism that is applied to all image pixels. As expected, the USM method and the implementation from Fig. 9] suffers from noise amplification and overshooting. 


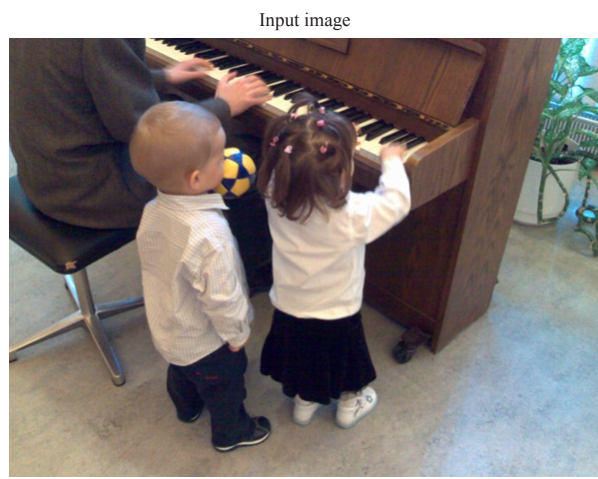

Input image

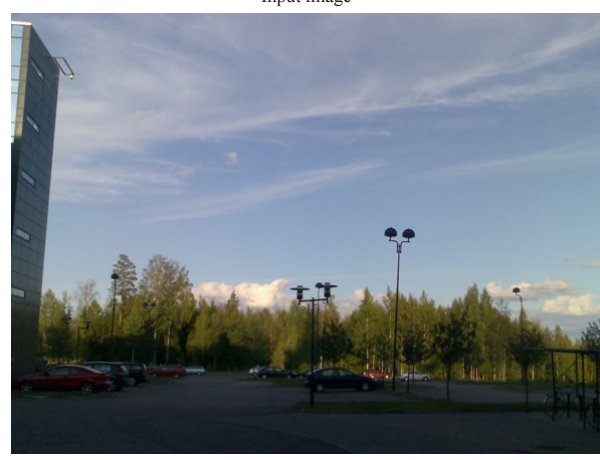

Result proposed

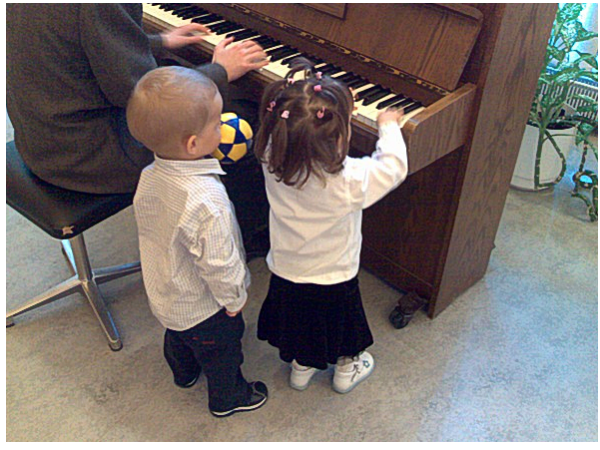

Result proposed

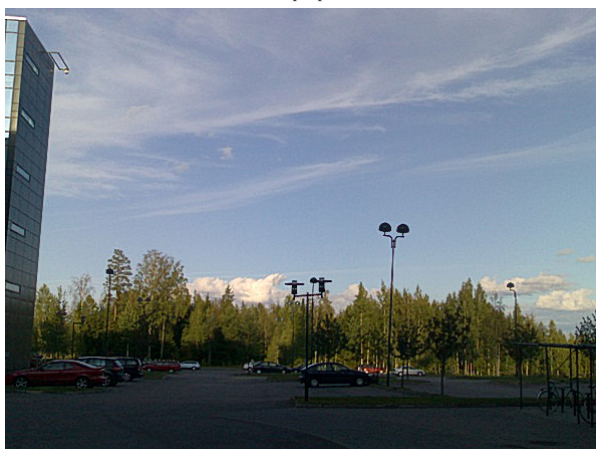

Fig. 15. Original image captured by a cameraphone (left column) and the result of the implementation from Fig. 10 (right column)

In the second set of experiments, several 8 bit digital color images, captured by different cameraphones and dedicated digital cameras, have been processed by the same enhancement methods and the results are shown in Fig. 12, Fig. 13 , Fig. 14 and Fig. 15. Again we can see that our proposed methods ensure more pleasant visual results with enhanced edges, reduced noise and no overshoots also for digital color images.

The sigma filter, used in our approach, necessitates the knowledge of the noise variance. In our implementation we have used the method from [2] to estimate the noise variance.

\section{Conclusions}

In this paper we have introduced a noise reduction and sharpening method suitable for mobile implementations. In our method we combined the sigma de-noising and the unsharp masking techniques through a simple clipping mechanism. We have seen that if clipping is done for all image pixels and not only for over enhanced edges the noise level in the processed image can be reduced 
together with increasing the edge sharpness. The proposed method can be combined with other existing approaches that adaptively adjusts the strength of sharpening based on the local image activity. Further developments in this direction as well as better clipping alternatives are currently under consideration and the results will be published elsewhere.

\section{References}

1. Bilcu, R.C., Vehvilainen, M.: A Novel Decomposition Scheme for Image De-noising. In: IEEE Int. Conf. on Ac. Speech and Sig. Proc., pp. 577-580 (2007)

2. Bilcu, R. C., Vehvilainen, M.: A New Method for Noise Estimation in Images. IEEE Int. Work. on Nonlin. Sig. and Im. Proc. (2005)

3. Fu, S., Ruan, Q., Wang, W.: A Coupled Shock-Diffusion Filter for Image Enhancement. In: IEEE Int. Conf. on Sig. Proc., pp. 16-20 (2006)

4. Lee, J.S.: Digital Image Smoothing and the Sigma Filter. Comp. Graph. Im. Proc. 24, 255-269 (1983)

5. Lukac, R., Plataniotis, K.: N.: Cost-Effective Sharpening of Single-Sensor Camera Images. In: IEEE Int. Conf. on Multimedia and Expo., pp. 829-832 (2006)

6. Matz, S.C., Figueiredo, R.J.P.: A Nonlinear Image Contrast Sharpening Approach Based on Munsell's Scale. IEEE Trans. on Im. Proc. 15(4), 900-909 (2006)

7. Mitra, K.S., Yu, T.-H.: Nonlinear Filters for Image Sharpening and Smoothing. IEEE Int. Conf. on Syst. Eng, 241-244 (1991)

8. Ojo, O., Kwaaitaal-Spassova, A., An Algorithm, T.G.: for Integrated Noise Reduction and Sharpness Enhancement. IEEE Trans. on Cons. El. 46(3), 474-480 (2000)

9. Polesel, A., Ramponi, G., Mathews, V.G.: Image Enhancement via Adaptive Unsharp Masking. IEEE Trans. on Im. Proc. 9(3), 505-510 (2000)

10. Russo, F.: An Image Enhancement Technique Combining Sharpening and Noise Reduction. IEEE Trans. on Instr. and Measur. 51(4), 824-828 (2002)

11. Rieder, P., Scheffer, G.: New Concepts on Denoising and Sharpening of Video Signals. IEEE Trans. on Cons. El. 47(3), 666-671 (2001)

12. Vehvilainen, M., Yrjanainen, J.: Circuit Arrangement to Accentuate the Sharpness and Attenuate the Noise of a Television Image. European Patent EP95101009 (1994)

13. Zhang, B., Allebach, J.: P.: Adaptive Bilateral Filter for Sharpness Enhancement and Noise Removal. In: IEEE Int. Conf. on Im. Proc., pp. VI417-VI420 (2007)

14. Wang, Q., Ward, R., Zou, J.: Contrast Enhancement for Enlarged Images Based on Edge Sharpening. IEEE Int. on Im. Proc. (2005) 